\title{
Chemokines as inflammatory mediators of fibrosis in liver fibrosis
}

\author{
H E Wasmuth \\ From 5th European Workshop on Immune-Mediated Inflammatory Diseases \\ Sitges-Barcelona, Spain. 1-3 December 2010
}

Chemokines are a class of small heparin-binding molecules which orchestrate the infiltration of leukocyte subsets into the liver during acute and chronic injury. In experimental models of liver fibrosis, chemokines are secreted by liver resident (e.g. hepatocytes, stellate cells) and liver infiltrating cells (e.g. leukocytes, platelets). Chemokines bind to characteristic G-protein coupled transmembraneous receptors which are also widely distributed within the liver.

Although the chemokine system has long been considered as having a high degree of redundancy, a number of important effects of specific chemokines and their receptors have been discovered within the last years. Notably, important chemokine effects beyond immune cells recruitment have also been identified.

In human hepatitis $C$ induced liver disease, the chemokine receptors CXCR3 and CCR5 are highly expressed on liver resident cells and on infiltrating lymphocytes. Both receptors have also been functionally studied in experimental models of liver injury.

CXCR3 binds the CXC chemokines CXCL9, CXCL10 and CXCL11. In a quantitative trait locus analysis in mice, these chemokines were shown to be located within a fibrosis associated haplotype block. Functional investigation of CXCL9 showed that this chemokine is secreted by hepatocytes and can bind to hepatic stellate cells through its receptor CXCR3. However, in contrast to most other chemokines, CXCL9 does not stimulate stellate cells, but represses the secretion and mRNA expression of collagen. As stellate cells are the pivotal collagen-producing cell types during liver fibrogenesis, these results implied antifibrotic properties of CXCL9. Indeed, mice lacking the CXCR3 receptor display pronounced liver fibrosis compared to their wild-type littermates after challenge with the hepatotoxins $\mathrm{CCl}_{4}$ and thioacetamide. In humans, a splice variant of CXCR3 can also bind the platelet-derived chemokine CXCL4. In contrast, the specific receptor for CXCL4 has not yet been identified in mice. However, CXCL4 can interact with other chemokines (e.g. CCL5) and thereby modulate their effects on target cells. In vivo, mice lacking CXCL4 are significantly protected from severe liver fibrosis, demonstrating that important effects of CXCL4 in mice are indeed mediated by other receptors than CXCR3. Amelioration of liver fibrosis in CXCL4 knockout mice is associated with lower pro-inflammatory chemokine levels (e.g. CCL5 and CXCL1) and a reduced infiltration of $\mathrm{T}$-cells and neutrophils into the liver. A similar reduction in infiltrating T-cells is observed in mice lacking the T-cell chemoattractant CCL5, which form heterodimers with CXCL4. In these mice, fibrosis is also greatly reduced in chronic damage models, which is in line with earlier findings about a pivotal role of the CCL5 receptors CCR1 and CCR5 in liver fibrosis.

Taken together, these results imply that chemokines play important, non-redundant roles during liver damage. Pharmacologically, chemokines and their receptors are amongst the best targetable molecules and the first chemokine receptor antagonists have already been licensed for human use in infectious diseases (mainly HIV infection). Thus, the further elucidation of specific chemokine pathways in liver diseases might lead to pre-clinical and clinical studies to modulate liver injury in the near future.

Published: 25 November 2010

doi:10.1186/1479-5876-8-S1-I13

Cite this article as: Wasmuth: Chemokines as inflammatory mediators of fibrosis in liver fibrosis. Journal of Translational Medicine 2010 8(Suppl 1):113. 